\section{Leiomyosarcoma of Renal Vein with Bone Metastasis: The Radiological Findings}

Sir,

Leiomyosarcoma (LMS) is a malignant mesenchymal tumour of smooth muscle origin. It represents $5-7 \%$ of soft tissue sarcomas. The diagnosis of primary renal vein LMS remains challenging because of its rarity. To the best of author's knowledge, this is the first case presented from Pakistan, though few cases have been reported in the international literature. We share an interesting case of a 50-year patient who had complaint of right hip pain for 2 months. The radiograph of the right hip joint showed an expansile osteolytic lesion in the iliac bone with cortical destruction and adjacent soft tissue density. MRI pelvis (Figure 1) showed a large T1 isointense, T2 hyper-intense, heterogeneously enhancing mass with hemorrhagic signal, necrotic areas, measuring $11.5 \times 11.3 \times 9.0 \mathrm{~cm}$ in CCxTVxAP dimensions, in the iliac bone extending to the acetabulum without intra-articular extension. It was infiltrating gluteus medius, minimus, obturator internus, with intrapelvic extension, and displacing sigmoid colon, and urinary bladder with intact fat planes. At this time, differentials were primary bone sarcoma versus metastatic deposits. Histopathology of the specimen showed spindled to oval shaped mediumsized cells with eosinophilic cytoplasm and markedly pleomorphic nuclei. Mitotic figures and foci of necrosis were noted. Immunohistochemical stains performed which showed ASMA positivity, favouring LMS. Contrast CT was done to rule out metastatic disease. No hepatic, pulmonary, or adrenal deposits were seen (Figure 2). However, it showed a heterogeneously enhancing mass lesion in the left renal vein (LRV) measuring $4.7 \times 4.8 \times$ $3.6 \mathrm{~cm}$, extending from renal hilum up to the insertion into inferior vena cava (IVC) and extending into one of the collateral veins along the left testicular vein at its insertion with retroperitoneal fat stranding. No extraluminal or IVC extension was seen. Left kidney showed normal vascularity without venous infarct. Multiple collaterals were seen in the perinephric area and also along the left testicular vein, bypassing the renal vein obstruction.

In 2017, Novak et al. studied the outcome and prognostic factors in the world case series of 67 patients with LMS of the renal vein. ${ }^{1}$ Aguilar et al. described a case report of LRV in a 76-year woman who underwent left radical nephrectomy with en-bloc resection of a para-aortic tumor, ${ }^{2}$ Microscopic examination showed moderately differentiated LMS. Later this patient demonstrated multiple metastasis to the liver.

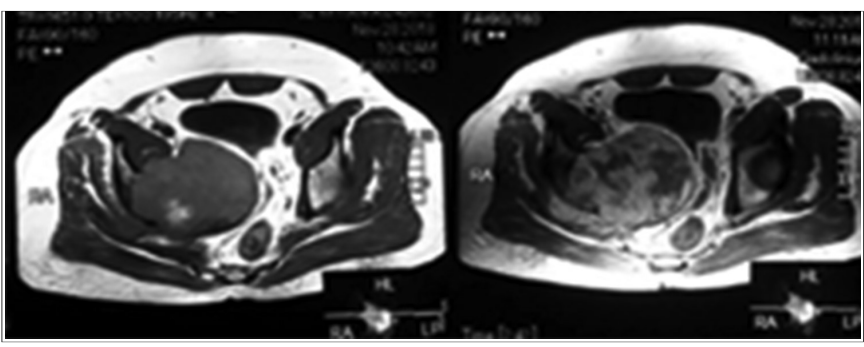

Figure 1: Contiguous axial plain and contrast T1 weighted sections of the MR pelvis showing right iliac bone metastasis.

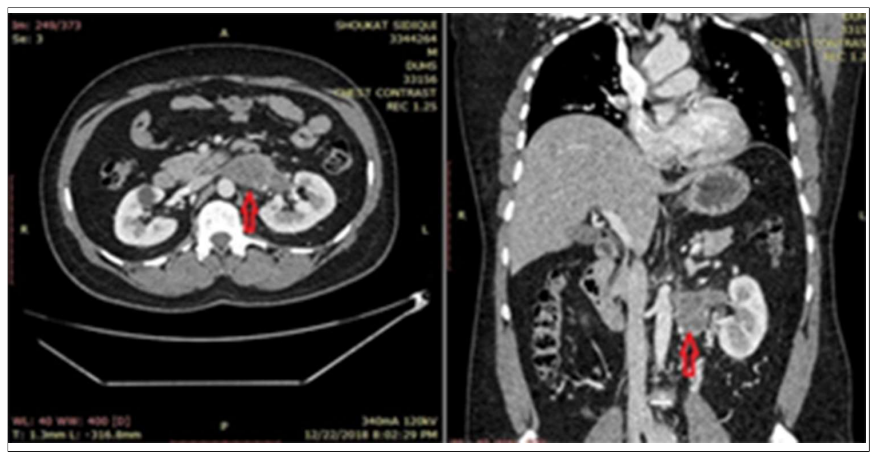

Figure 2: Contiguous axial and coronal post contrast CT images of the abdomen showing left renal vein mass.

Lipton et al. reported a case of a patient with a large left flank mass, diagnosed clinically as renal cell carcinoma. ${ }^{3}$ The combination of CT, ultrasound, and angiographic studies suggested a tumor arising from the renal vein with enlarged kidney. The patient underwent surgery; and histology showed high-grade LMS.

This case highlights the importance of radio pathological correlation with relevant multimodality imaging including X-ray, MR, CT imaging, CT-guided biopsy in the diagnosis of rare diseases. As this patient had metastatic disease, he was referred to oncology department for further management.

\section{CONFLICT OF INTEREST:}

Author declared no conflict of interest.

\section{AUTHOR'S CONTRIBUTION:}

$\mathrm{MH}$ : Made substantial contributions to the design of the work; drafted the work and revised it critically for important intellectual content.

\section{REFERENCES}

1. Novak M, Perhavec A, Maturen K, Djokic SP, Jereb S, Erzen D. Leiomyosarcoma of the renal vein: Analysis of outcome and prognostic factors in the world case series of 67 patients. Radiol Oncol 2017; 51:56-64.

2. Aguilar IC, Benavente VA, Pow-Sang MR, Morante CM, Meza L, Destefano $\mathrm{V}$, et al. Leiomyosarcoma of the renal vein: Case report and review of the literature. Urol Oncol 2005; 23:22-6. 
3. Lipton ML, Sprayregen S, Kutcher R, Frost A. Venous invasion in renal vein leiomyosarcoma: Case report and review of the literature. Abdom Imaging 1995; 20:64-7.

Mahnoor Hafeez

Department of Radiology, Dow Institute of Radiology, Dow University of Health Sciences (DUHS), Karachi, Pakistan
Correspondence to: Dr. Mahnoor Hafeez, Department of Radiology, Dow Institute of Radiology, Dow University of Health Sciences (DUHS), Karachi, Pakistan

E-mail:mahnoor.hafeez@yahoo.com

Received: January 09, 2019; Revised: February 21, 2019; Accepted: February 21, 2019

…ㄴ..... 